\title{
A Comparative study of Plasma-treated Oxygen- doped Single and Multiwalled Carbon Nanotubes as Electrocatalyst for Efficient Oxygen Reduction Reaction
}

Roopathy Mohan, Arindam Modak and Alex Schechter*

Department of Chemical Sciences, Ariel University, Ariel research park 40700, Israel

*E-mail for correspondence: salex@ariel.ac.il

Phone: +97239371470, Fax: +97239076586

Number of Pages: 13 (including the cover sheet)

Number of Figures: 9

Number of Tables: 7 

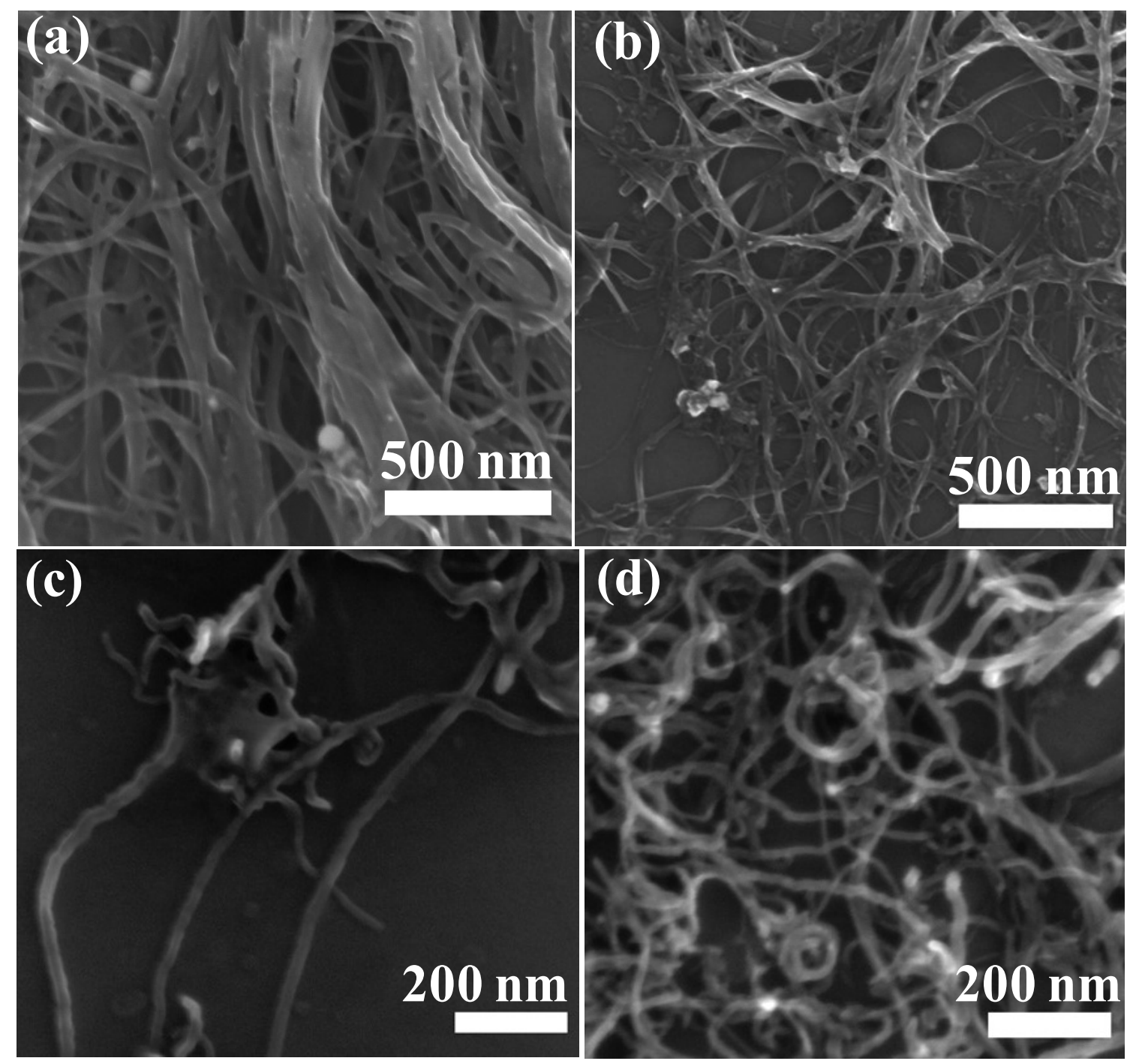

Figure S1. SEM image of (a) SWCNT untreated (b) SWCNT $20 \mathrm{~min} \mathrm{O}_{2}$ plasma treated, (c) MWCNT untreated and (d) MWCNT 20 min $\mathrm{O}_{2}$ plasma treated. 

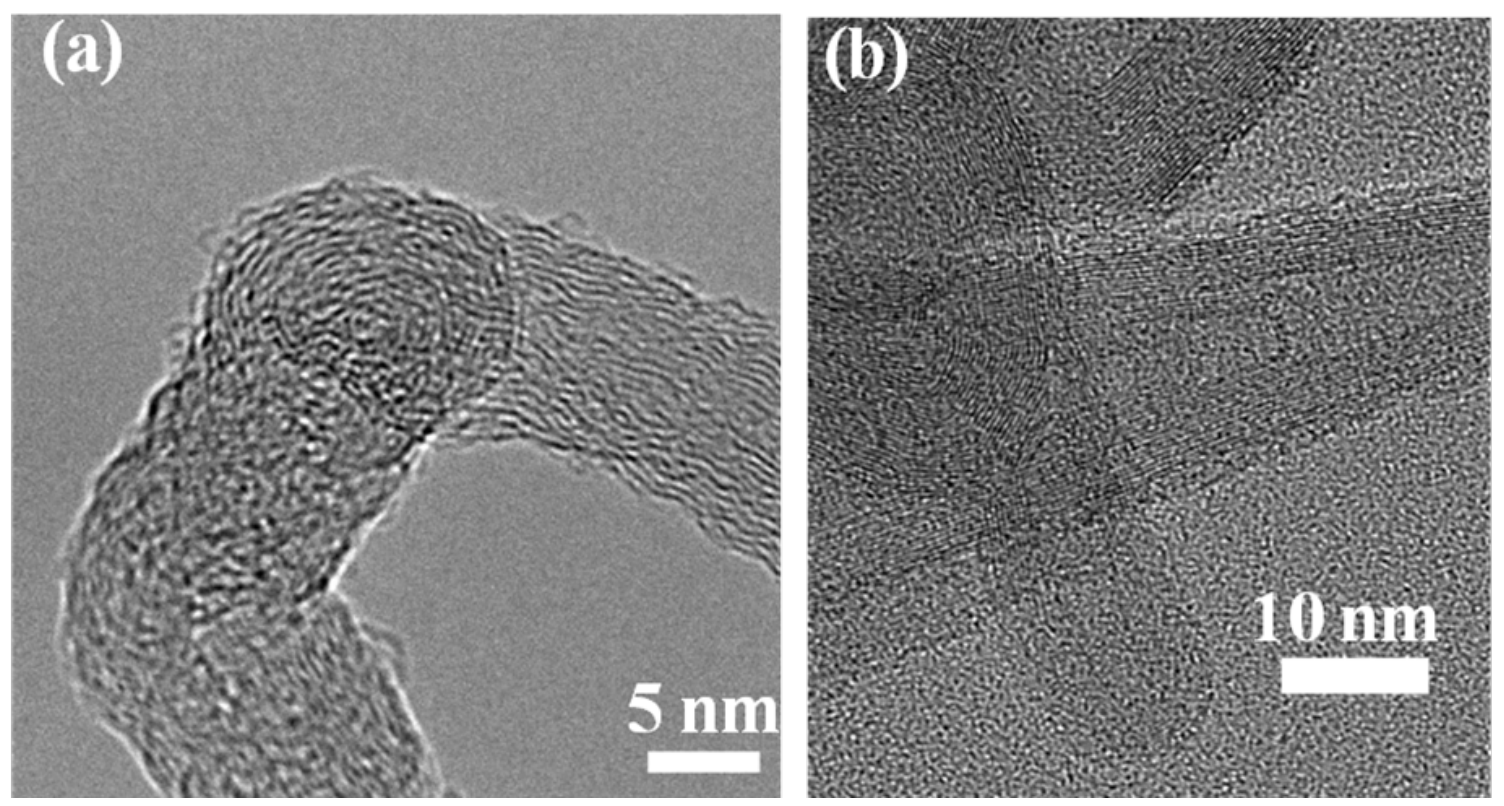

Figure S2. HR-TEM image of plasma treated SWCNT (a) and MWCNT (b) 


\section{(a)}
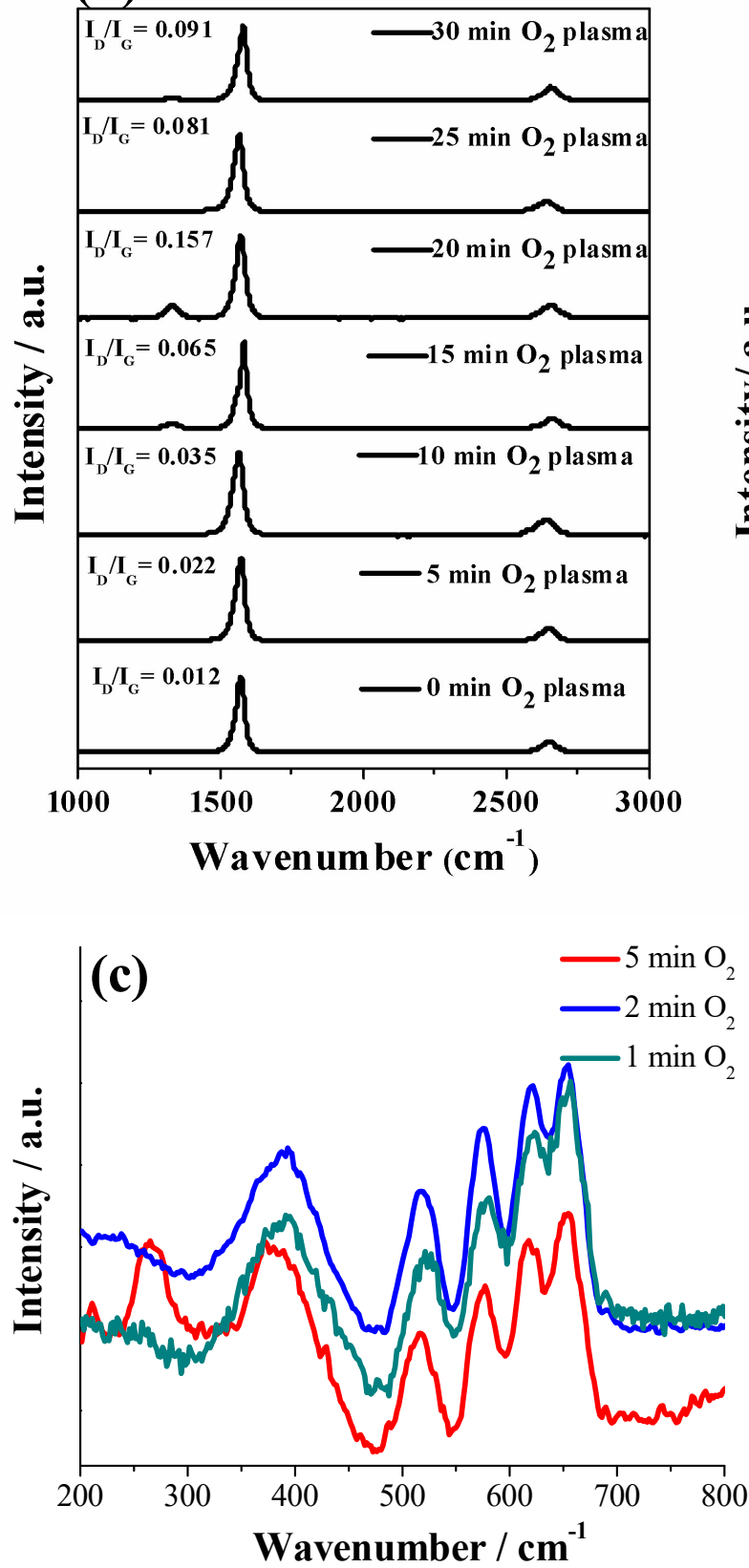

(b)
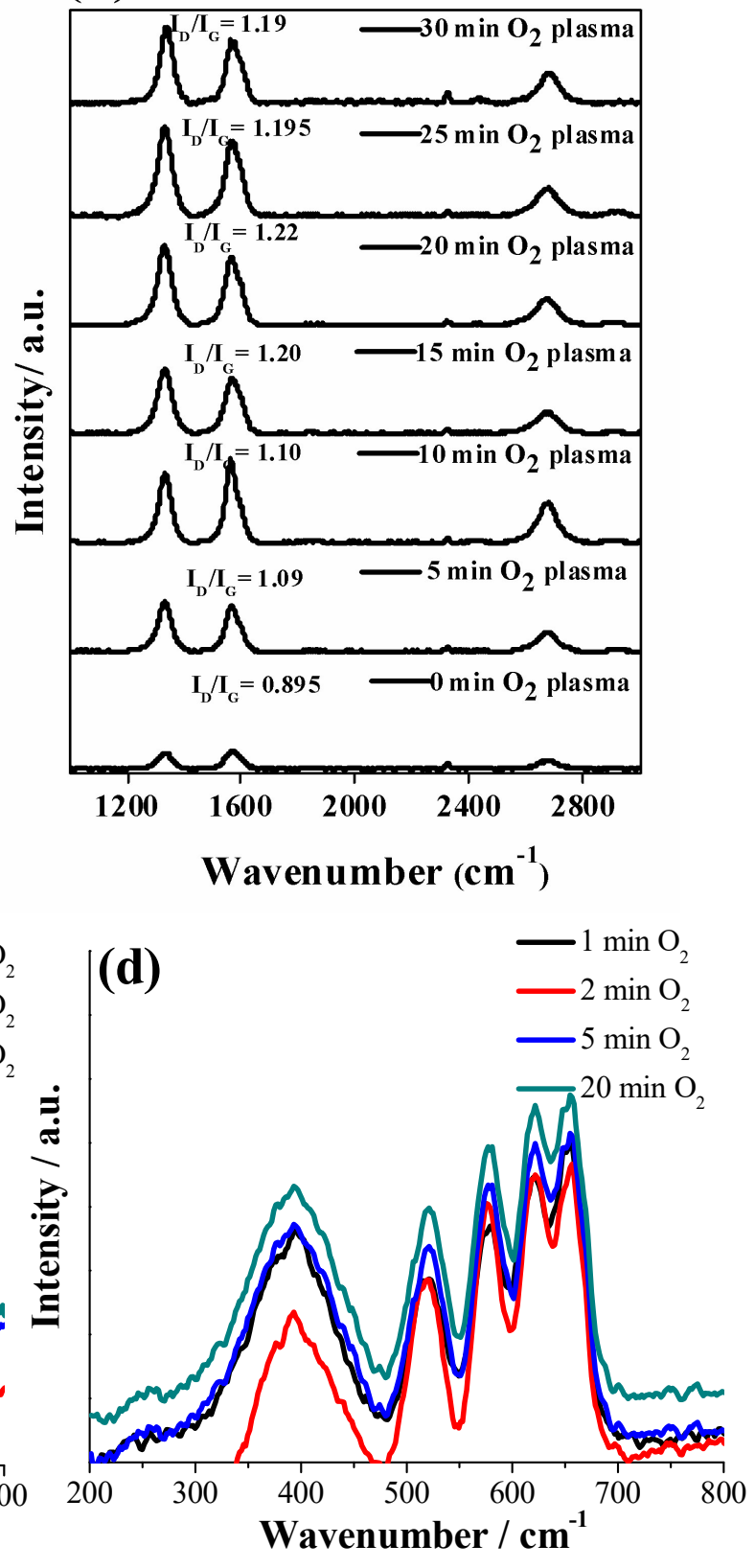

Figure S3. Raman spectra of G and D band zone for plasma treated (a, c) single walled and (b, d) multiwalled CNTs at different plasma exposure time. 

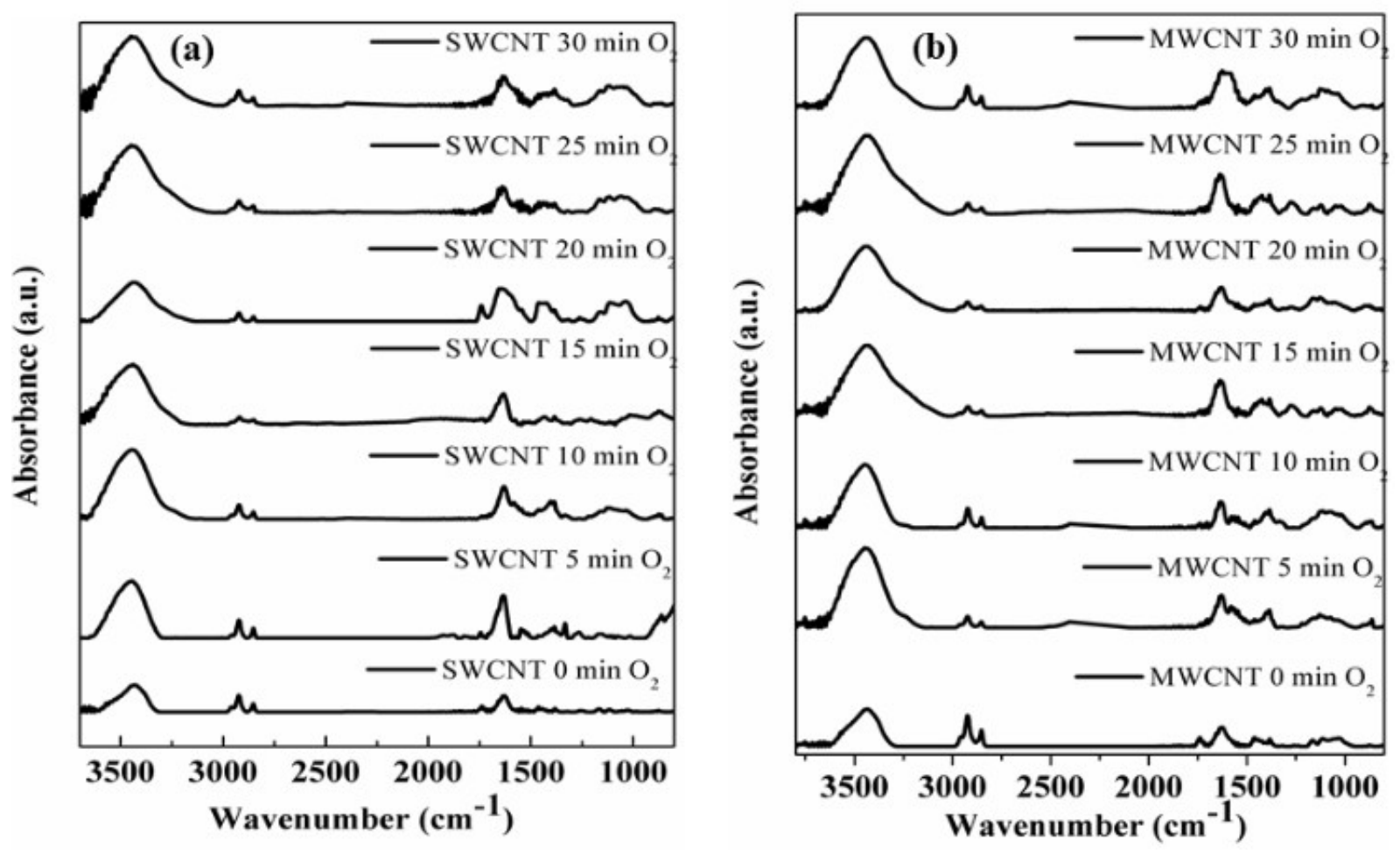

Figure S4.-FT-IR spectra of pristine and plasma treated (a) SWCNT and (b) MWCNT at various plasma exposure time. 


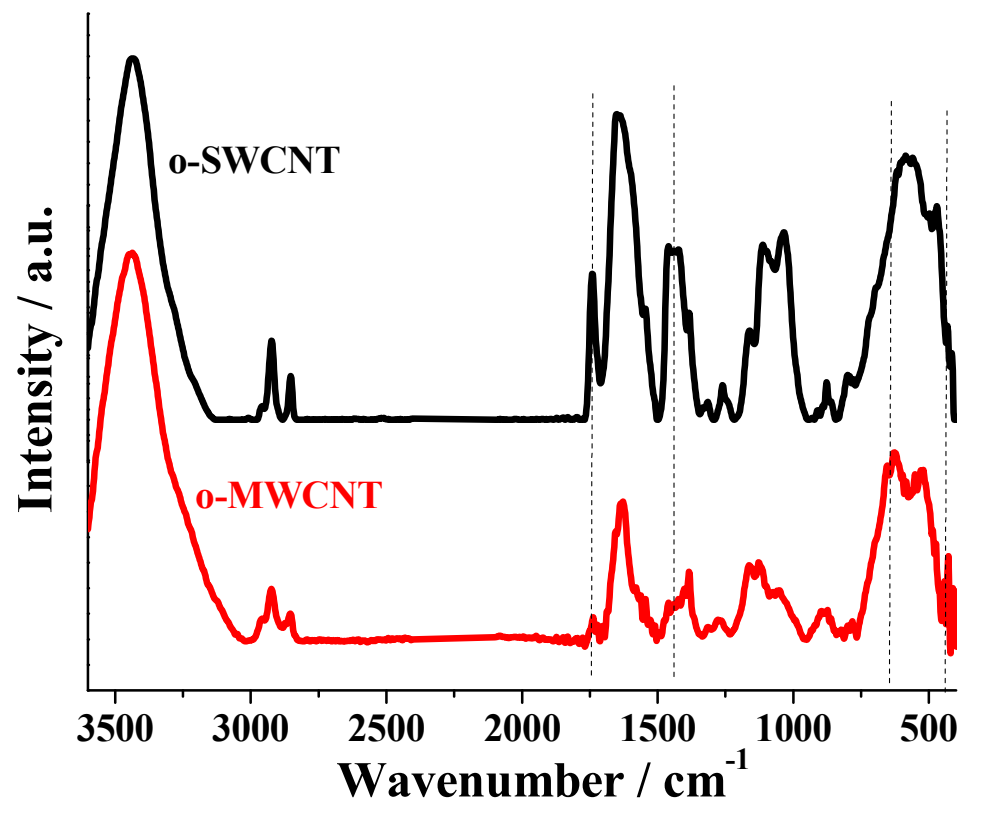

Figure S5. FT-IR spectra of $\mathrm{O}_{2}$ plasma functionalized (a) SWCNT and (b) MWCNT. 
Table S1. Absorption band integration of untreated and different plasma time treated FT-IR analysis

\begin{tabular}{|c|c|c|c|c|c|c|c|c|}
\hline \multirow{2}{*}{$\begin{array}{c}\text { Plasma } \\
\text { time } \\
\text { (min) }\end{array}$} & \multicolumn{2}{|c|}{-OH stretch } & \multicolumn{2}{|c|}{-CH stretch } & \multicolumn{2}{|c|}{$-C=O$ stretch } & \multicolumn{2}{|c|}{$-C=C$ stretch } \\
\hline & SWCNT & MWCNT & SWCNT & MWCNT & SWCNT & MWCNT & SWCNT & MWCNT \\
\hline 0 & 0.086 & 0.717 & 1.695 & 1.476 & 0.382 & 0.472 & 0.026 & 0.324 \\
\hline 5 & 1.059 & 0.791 & 0.98 & 0.389 & 0.325 & 0.363 & 0.057 & 0.034 \\
\hline 10 & 1.775 & 0.832 & 0.863 & 0.345 & 0.394 & 0.42 & 0.225 & 0.12 \\
\hline 15 & 2.992 & 0.986 & 0.623 & 0.303 & 0.424 & 0.48 & 0.347 & 0.19 \\
\hline 20 & 3.317 & 2.695 & 0.452 & 0.271 & 1.801 & 1.796 & 0.201 & 0.12 \\
\hline 25 & 2.983 & 2.077 & 0.532 & 0.41 & 0.702 & 0.543 & 0.237 & 0.116 \\
\hline 30 & 1.862 & 1.946 & 0.693 & 0.484 & 0.386 & 0.427 & 0.265 & 0.181 \\
\hline
\end{tabular}

Table S2. CHNO elemental analysis of SWCNT and MWCNT

\begin{tabular}{|c|c|c|c|c|}
\hline Sample name & $\begin{array}{c}\text { Nitrogen } \\
\text { wt } \% \\
\end{array}$ & $\begin{array}{c}\text { Carbon } \\
\text { wt } \% \\
\end{array}$ & $\begin{array}{c}\text { Hydrogen } \\
\text { wt\% } \\
\end{array}$ & $\begin{array}{c}\text { Oxygen } \\
\text { wt } \% \\
\end{array}$ \\
\hline SWCNT & 0.18 & 99.72 & 0.08 & 1.33 \\
\hline SWCNT 5 min $\mathrm{O}_{2}$ & 0.2 & 97.2 & 0.15 & 2.43 \\
\hline SWCNT $10 \min \mathrm{O}_{2}$ & 0.21 & 95.76 & 0.2 & 3.82 \\
\hline SWCNT $15 \min \mathrm{O}_{2}$ & 0.2 & 95.66 & 0.27 & 3.87 \\
\hline SWCNT 20 min $\mathrm{O}_{2}$ & 0.17 & 94.58 & 0.35 & 4.88 \\
\hline SWCNT $25 \min \mathrm{O}_{2}$ & 0.17 & 96.02 & 0.2 & 3.6 \\
\hline SWCNT $30 \min \mathrm{O}_{2}$ & 0.17 & 95.93 & 0.26 & 3.4 \\
\hline MWCNT & 0 & 99.52 & 0.08 & 0.36 \\
\hline MWCNT $5 \min \mathrm{O}_{2}$ & 0 & 98.54 & 0.11 & 0.37 \\
\hline MWCNT $10 \min \mathrm{O}_{2}$ & 0 & 98.22 & 0.12 & 1.65 \\
\hline MWCNT $15 \min \mathrm{O}_{2}$ & 0 & 97.99 & 0.11 & 1.89 \\
\hline MWCNT $20 \min \mathrm{O}_{2}$ & 0 & 97.51 & 0.14 & 2.33 \\
\hline MWCNT $25 \min \mathrm{O}_{2}$ & 0 & 97.9 & 0.18 & 1.81 \\
\hline MWCNT $30 \min \mathrm{O}_{2}$ & 0 & 98.26 & 0.12 & 1.61 \\
\hline
\end{tabular}


A complementary, C-H-N-O elemental analysis was carried out to determine the total oxygen content percentage after different exposure time of O-SWCNT and O-MWCNT (Table S2). The obtained C/O ratio of SWCNT and MWCNT material decreased from untreated samples i.e. 74.49 to 19.38 and from 256.66 to 41.69 respectively after 20 minutes exposure. It should be remembered that CHNO elemental technique provides a total analysis view of the bulk. In our study it clearly proves that 20 minutes treatment enriches the CNT samples with oxygen. In table S2, the gradual increase in trend of hydrogen and oxygen are clearly seen from untreated to 20 min plasma exposed SWCNT and MWCNT, above 20 minutes trend gradually decreased. Similarly increase in $\mathrm{H}$ and $\mathrm{O}$ content leads to destruction of C content. Hence proves that defective carbon is more in 20 minutes plasma treatment compare to pristine and other exposure time.

Table S3. Quantification of deconvoluted C1s spectra (\%)

\begin{tabular}{|c|c|c|c|c|c|c|c|}
\hline Catalyst & $\begin{array}{c}C=C \\
284.5 \mathrm{eV}\end{array}$ & $\begin{array}{c}\mathrm{C}-\mathrm{C} \\
285.2 \mathrm{eV}\end{array}$ & $\begin{array}{c}\text { C-O } \\
286.0 \\
\text { eV }\end{array}$ & $\begin{array}{c}\text { C-O-C } \\
286.9 \\
\text { eV }\end{array}$ & $\begin{array}{c}-\mathrm{C}=\mathrm{O} \\
\mathrm{O}-\mathrm{C}-\mathrm{O} \\
287.8 \mathrm{eV}\end{array}$ & $\begin{array}{c}-\mathrm{COO} \\
288.7 \mathrm{eV}\end{array}$ & $\begin{array}{c}\mathrm{COO} \\
\mathrm{CO}_{3} \\
289.6 \mathrm{eV}\end{array}$ \\
\hline $\begin{array}{l}\text { SWCNT } \\
\text { untreated }\end{array}$ & 54.9 & 11.80 & 8.00 & 4.57 & 2.96 & 2.41 & 1.06 \\
\hline $\begin{array}{l}20 \mathrm{~min} \mathrm{O}_{2} \\
\text { plasma } \\
\text { treated } \\
\text { SWCNT } \\
\end{array}$ & 53.2 & 12.00 & 7.60 & 7.05 & 4.39 & 2.60 & 1.09 \\
\hline $\begin{array}{l}\text { MWCNT } \\
\text { untreated }\end{array}$ & 54.2 & 12.00 & 7.70 & 4.41 & 2.69 & 2.15 & 1.17 \\
\hline $\begin{array}{l}20 \mathrm{~min} \mathrm{O}_{2} \\
\text { plasma } \\
\text { treated } \\
\text { MWCNT }\end{array}$ & 44.2 & 12.49 & 7.00 & 12.58 & 4.69 & 4.07 & 1.97 \\
\hline
\end{tabular}


Table S4. Quantification of deconvoluted O1s spectra (\%)

\begin{tabular}{|c|c|c|}
\hline Catalyst & $\begin{array}{c}-\mathrm{OH}, \mathrm{O}-\mathrm{C}-\mathrm{O}_{\mathrm{cyc}} \\
533.2 \mathrm{eV}\end{array}$ & $\begin{array}{c}-\mathrm{C}=\mathrm{O}, \mathrm{O}-\mathrm{C}-\mathrm{O}_{\mathrm{cyc},}-\mathrm{COO} \\
531.7 \text { to } 532.7 \mathrm{eV}\end{array}$ \\
\hline SWCNT untreated & 32.5 & 45.57 \\
\hline $\begin{array}{l}20 \text { min } \mathrm{O}_{2} \text { plasma } \\
\text { treated SWCNT }\end{array}$ & 44.6 & 26.99 \\
\hline MWCNT untreated & 1.74 & 91.72 \\
\hline $\begin{array}{l}20 \text { min } \mathrm{O}_{2} \text { plasma } \\
\text { treated MWCNT }\end{array}$ & 1.74 & 92.82 \\
\hline
\end{tabular}
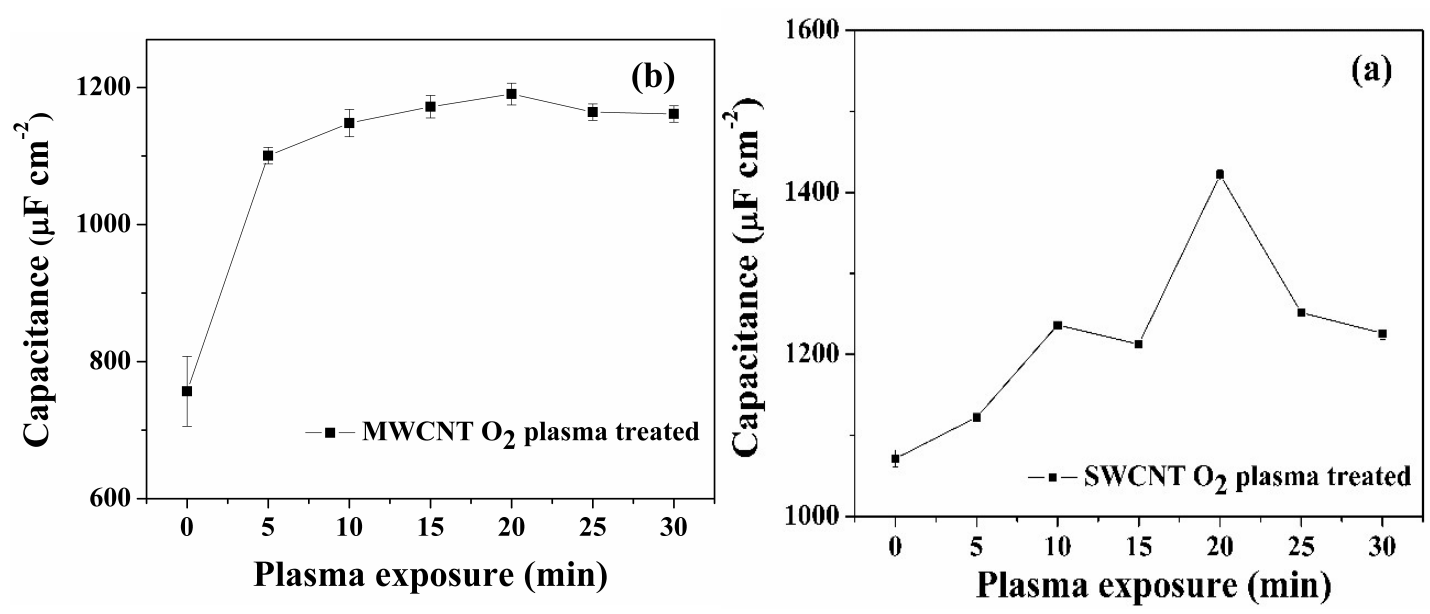

Figure S6. (a) Capacitance calculated from SWCNT unfunctionalized and O-SWCNT at different plasma exposure times; (b) Capacitance calculated from MWCNT unfunctionalized and O-MWCNT at different plasma exposure time 

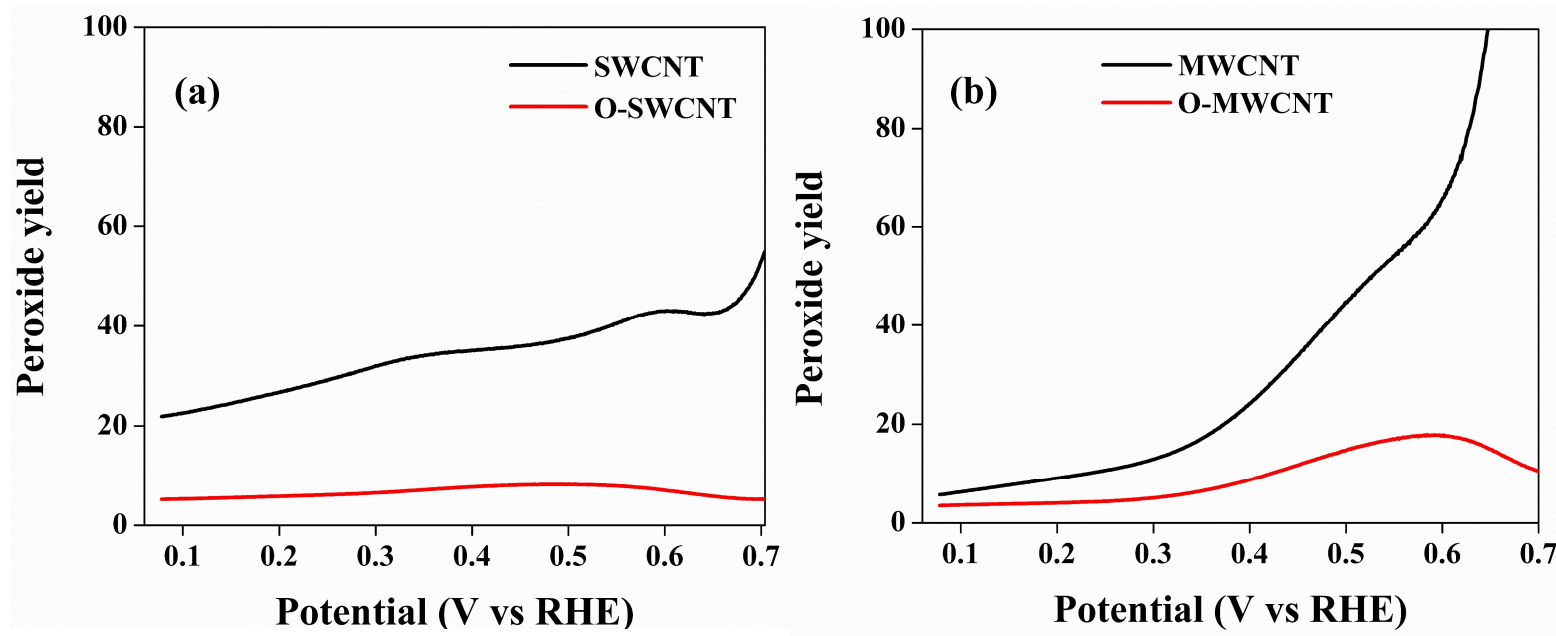

Figure S7. Hydrogen peroxide yield of pristine and oxygen plasma treated (a) SWCNT and (b) MWCNT

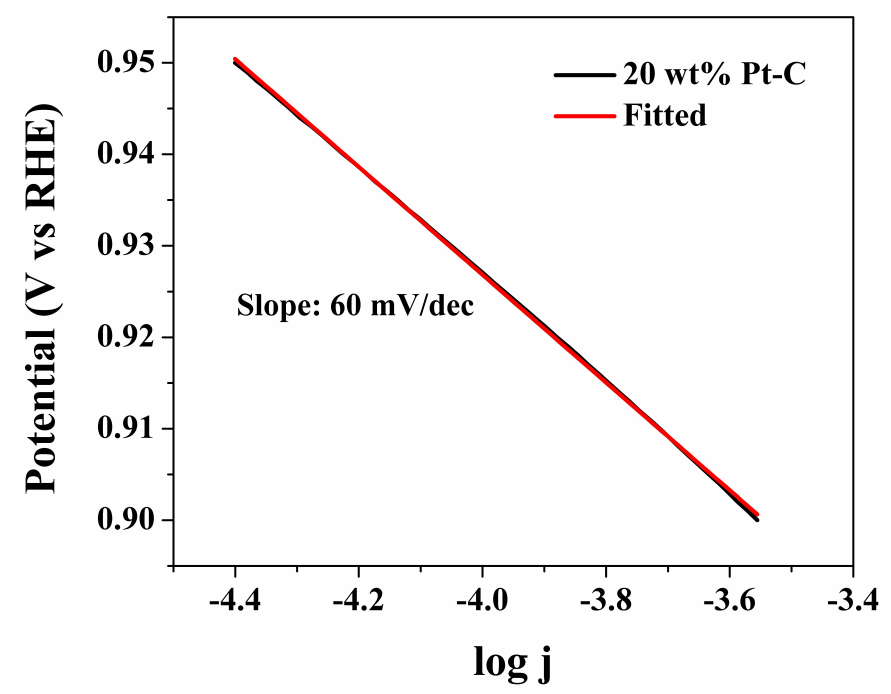

Figure S8. Tafel plot of commercial $20 \mathrm{wt} \% \mathrm{Pt}-\mathrm{C}$ 
Table S5. ORR activity of SWCNT and oxygen plasma treated SWCNT (Note: O-Oxygen plasma treated)

\begin{tabular}{|c|c|c|c|c|}
\hline Catalyst & $\begin{array}{c}\text { Onset } \\
\text { potential } \\
\text { V vs RHE }\end{array}$ & $\begin{array}{c}\text { Current } \\
\text { density at } 0.2 \\
\text { V vs RHE } \\
\left(\mathrm{mA} \mathrm{cm} \mathrm{cm}^{-2}\right)\end{array}$ & $\begin{array}{c}\mathrm{HO}_{2}^{-} \text {Ring current } \\
\text { density at } 0.5 \mathrm{~V} \text { vs } \\
\mathrm{RHE} \\
\left.(\mathrm{mA} \mathrm{cm})^{-2}\right)\end{array}$ & $\begin{array}{c}\text { Capacitance } \\
(\mu \mathrm{F})\end{array}$ \\
\hline SWCNT untreated & 0.74 & -4.17 & 0.018 & 210 \\
\hline O-SWCNT $5 \mathrm{~min}$ & 0.75 & -4.08 & 0.005 & 220 \\
\hline O-SWCNT $10 \mathrm{~min}$ & 0.76 & -3.43 & 0.012 & 242 \\
\hline O-SWCNT 15 min & 0.76 & -3.02 & 0.011 & 236 \\
\hline O-SWCNT $20 \mathrm{~min}$ & 0.78 & -3.7 & 0.003 & 278 \\
\hline O-SWCNT 25 min & 0.76 & -3.44 & 0.004 & 247 \\
\hline O-SWCNT $30 \mathrm{~min}$ & 0.77 & -3.84 & 0.007 & 242 \\
\hline
\end{tabular}

Table S6. ORR activity of MWCNT and oxygen plasma treated MWCNT (Note: O-Oxygen plasma treated)

\begin{tabular}{|c|c|c|c|c|}
\hline Catalyst & $\begin{array}{l}\text { Onset potential } \\
\text { V vs RHE }\end{array}$ & 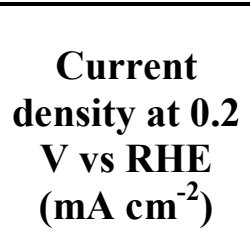 & $\begin{array}{c}\mathrm{HO}_{2}^{-} \text {Ring current } \\
\text { density at } 0.5 \mathrm{~V} \text { vs } \\
\mathrm{RHE} \\
\left.(\mathrm{mA} \mathrm{cm})^{2}\right)\end{array}$ & $\begin{array}{c}\text { Capacitance } \\
(\mu \mathrm{F})\end{array}$ \\
\hline MWCNT untreated & 0.74 & -2.34 & 0.032 & 95 \\
\hline O-MWCNT $5 \mathrm{~min}$ & 0.75 & -2.71 & 0.022 & 138 \\
\hline O-MWCNT $10 \mathrm{~min}$ & 0.75 & -3.11 & 0.018 & 144 \\
\hline O-MWCNT 15 min & 0.76 & -2.98 & 0.016 & 147 \\
\hline O-MWCNT 20 min & 0.78 & -3.95 & 0.006 & 150 \\
\hline O-MWCNT $25 \mathrm{~min}$ & 0.76 & -3.68 & 0.009 & 146 \\
\hline O-MWCNT $30 \mathrm{~min}$ & 0.76 & -2.88 & 0.013 & 146 \\
\hline
\end{tabular}



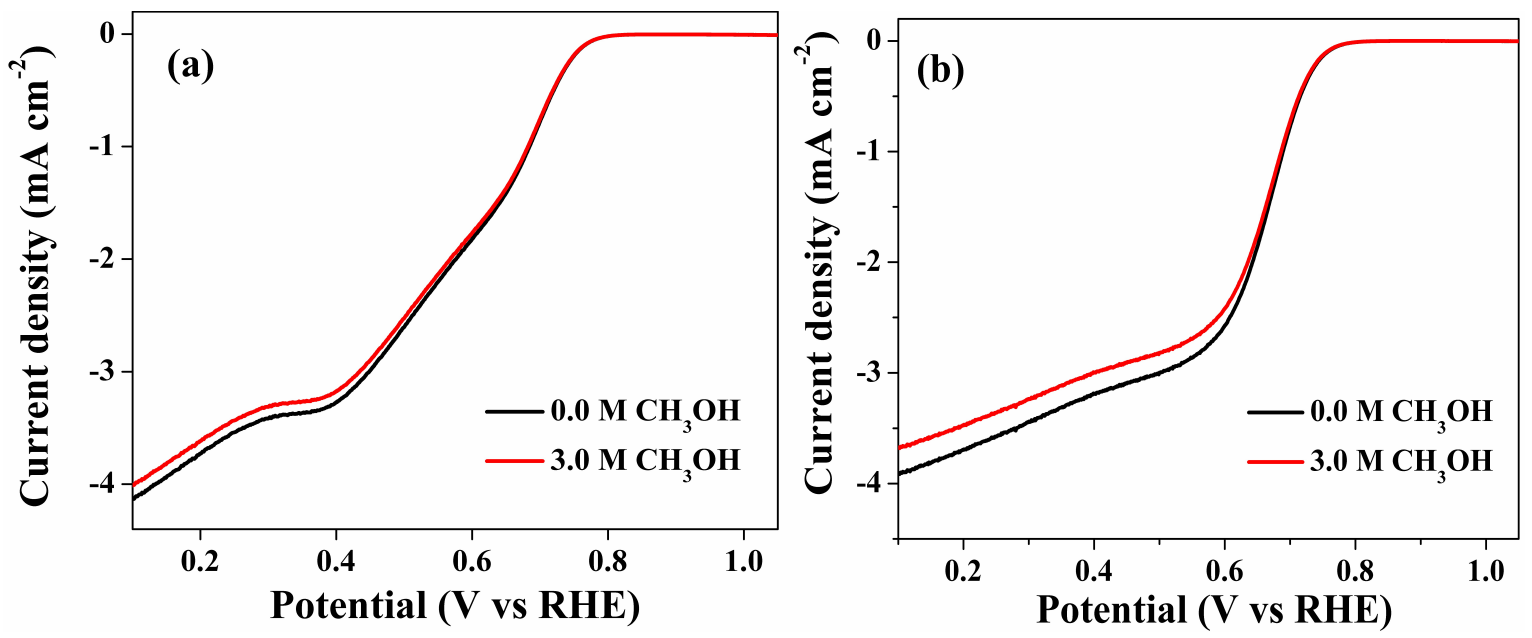

Figure S9. Methanol tolerance test of (a) O-SWCNT and (b) O-MWCNT

Table S7. Comparative study between O-SWCNT and other metal free and nitrogen free catalyst forORR ${ }^{1-8}$

\begin{tabular}{|l|l|c|}
\hline Catalyst & Onset potential & Ref \\
\hline $\begin{array}{l}\alpha-\mathrm{Fe}_{2} \mathrm{O}_{3} / \mathrm{N}- \\
\mathrm{CNTs}\end{array}$ & $-0.21 \mathrm{~V}$ vs Ag/AgCl & 1 \\
\hline $\mathrm{h}-\mathrm{CNT} 500-2 \mathrm{~h}$ & $-0.184 \mathrm{~V}$ vs Ag/AgCl & 2 \\
\hline Fe-N-HOPG & $0.75 \mathrm{~V}$ vs RHE & 3 \\
\hline $\mathrm{N}-\mathrm{SWNT}-2$ & $-0.35 \mathrm{~V}$ vs NHE & 4 \\
\hline SWCNT/N-GS & $0.75 \mathrm{~V}$ vs RHE & 5 \\
\hline MWCNT/N-GS & $0.61 \mathrm{~V}$ vs RHE & 7 \\
\hline P-CC & $0.76 \mathrm{~V}$ vs RHE & 8 \\
\hline BCNP & $-0.18 \mathrm{~V}$ vs Ag/AgCl & This work \\
\hline IGnP & $-0.14 \mathrm{~V}$ vs Ag/AgCl & \\
\hline O-SWCNT and & $0.78 \mathrm{~V}$ vs RHE & \\
\hline O-MWCNT & & \\
\hline
\end{tabular}




\section{References}

1. Sun, M.; Zhang, G.; Liu, H.; Liu, Y.; Li, J. $\alpha$ - and $\gamma$-Fe2O3 Nanoparticle/Nitrogen Doped Carbon Nanotube Catalysts for High-Performance Oxygen Reduction Reaction. Sci. China Mater. 2015, 58 (9), 683-692, DOI 10.1007/s40843-015-0082-x.

2. Liu, M.; Li, J. Heating Treated Carbon Nanotubes As Highly Active Electrocatalysts for Oxygen Reduction Reaction. Electrochim. Acta 2015, 154, 177-183, DOI 10.1016/j.electacta.2014.12.039.

3. Kolagatla, S.; Subramanian, P.; Schechter, A. Nanoscale Mapping of Catalytic Hotspots on Fe, N-Modified HOPG by Scanning Electrochemical Microscopy-Atomic Force Microscopy. Nanoscale 2018, 10 (15), 6962-6970, DOI 10.1039/C8NR00849C.

4. Lu, D.; Wu, D.; Jin, J.; Chen, L. Defect-Induced Catalysis toward the Oxygen Reduction Reaction in Single-Walled Carbon Nanotube: Nitrogen Doped and Non-Nitrogen Doped. Electrochim. Acta 2016, 215, 66-71, DOI 10.1016/j.electacta.2016.08.082.

5. Fujigaya, T.; Morita, J.; Nakashima, N. Grooves of Bundled Single-Walled Carbon Nanotubes Dramatically Enhance the Activity of the Oxygen Reduction Reaction. ChemCatChem 2014, 6 (11), 3169-3173, DOI 10.1002/cctc.201402565.

6. Liu, Z.; Zhao, Z.; Wang, Y.; Dou, S.; Yan, D.; Liu, D.; Xia, Z.; Wang, S. In Situ Exfoliated, Edge-Rich, Oxygen-Functionalized Graphene from Carbon Fibers for Oxygen Electrocatalysis. Adv. Mater. 2017, 29 (18), 1606207, DOI 10.1002/adma.201606207.

7. Panomsuwan, G.; Saito, N.; Ishizaki, T. Electrocatalytic Oxygen Reduction Activity of BoronDoped Carbon Nanoparticles Synthesized via Solution Plasma Process. Electrochem. commun. 2015, 59, 81-85, DOI 0.1016/j.elecom.2015.07.005.

8. Jeon, I.-Y.; Choi, H.-J.; Choi, M.; Seo, J.-M.; Jung, S.-M.; Kim, M.-J.; Zhang, S.; Zhang, L.; Xia, Z.; Dai, L.; et al. Facile, Scalable Synthesis of Edge-Halogenated Graphene Nanoplatelets as Efficient Metal-Free Eletrocatalysts for Oxygen Reduction Reaction. Sci. Rep. 2013, 3 (1), 1810, DOI 10.1038/srep01810. 